\section{Obstrução de via aérea superior secundária a cisto epidermóide laringiano em gato: relato de caso}

\author{
Upper airway obstruction secondary to laryngeal epidermoid \\ cyst in cat: case report
}

Bruno Ricardo Soares Alberigi da Silva*, Mário dos Santos Filho², Alexandre José Rodrigues Bendas ${ }^{3}$, Denise do Vale Soares ${ }^{4}$, Rebeca Guimaraes Moraes Rego Bhering Mattos ${ }^{4}$, Sara Maria de Carvalho e Suzano ${ }^{5}$ \& Jonimar Pereira Paiva ${ }^{6}$

'Médico veterinário, MSc, Doutorando. Programa de Pós-graduação em Medicina Veterinária - Ciências Clínicas, Instituto de Veterinária, Universidade Federal Rural do Rio de Janeiro - UFRRJ, Seropédica, RJ, Brasil

2Médico veterinário, Mestrando. Programa de Pós-graduação em Medicina Veterinária - Ciências Clínicas, Instituto de Veterinária, Universidade Federal Rural do Rio de Janeiro - UFRRJ, Seropédica, RJ, Brasil

3Médico veterinário, MSc, Doutorando. Programa de Pós-graduação em Medicina Veterinária - Clínica e Reprodução Animal, Faculdade de Veterinária, Universidade Federal Fluminense - UFF, Niterói, RJ, Brasil

${ }^{4}$ Médicas veterinárias, Autônomas. Instituto de Especialidades em Medicina Veterinária - IEMEV, Rio de Janeiro, RJ, Brasil

${ }_{5}^{5}$ Médica veterinária, DSc. Escola de Veterinária, Universidade Castelo Branco - UCB, Rio de Janeiro, RJ, Brasil

${ }^{6}$ Médico veterinário, DSc. Departamento de Medicina e Cirurgia Veterinária, Instituto de Veterinária, Universidade Federal Rural do Rio de Janeiro - UFRRJ, Seropédica, RJ, Brasil

\section{Resumo}

As doenças de laringe são raras em gatos, porém quando presentes podem causar dispneia grave, comprometendo o aporte ideal de oxigênio para o organismo. Foi atendido um paciente da espécie felina, fêmea, de 15 anos de idade, sem raça definida, em quadro de dispneia mista. Com os achados de exame físico e exames complementares, suspeitou-se de neoplasia em região cervical obstruindo as vias aéreas anteriores. O paciente foi a óbito, sendo realizada necropsia que revelou estrutura de formato arredondado e aspecto liso e brilhante em região da laringe, projetando-se para luz do órgão, sugerindo ser a causa do comprometimento da passagem do fluxo de ar. A análise histopatológica concluiu que se tratava de uma neoformação benigna. O presente relato tem como objetivo descrever um caso de cisto epidermóide laringiano, causando obstrução de via aéreas anteriores em um gato, com seus achados de exame físico e de exames de imagem como apoio diagnóstico.

Palavras-chave: felino, dispneia, laringe.

\begin{abstract}
Laryngeal diseases are rare in cats, but when present they can cause significant dyspnea, compromising the ideal supply of oxygen to the body. A female cat, of 15 years old, mixed breed, was attended in dyspnea state. With findings of physical examination and complementary exams cervical neoplasia was suspected to obstruct the upper airways. After the histopathological analysis of the mass found in the region of the larynx, it was concluded that it was a benign mass that compromised the flow of air through the larynx. The present work aims to report a case of a laryngeal epidermoid cyst causing upper airway obstruction in a cat, describing its physical examination findings and imaging as diagnostic support.
\end{abstract}

Keywords: feline, dyspnea, larynx.

\section{Introdução}

As doenças de laringe são raras em gatos, porém quando presentes podem causar dispneia grave (Corgozinho et al., 2008; Macphail, 2014). Em um estudo retrospectivo a afecção de laringe mais frequente foi a paralisia (40\%), seguida por neoplasias (29\%), afecções inflamatórias (17\%) e outras causas (14\%) como, por exemplo, cistos e alterações de etiologia não definida (Taylor et al., 2009).

Cistos epidermóides são lesões císticas originadas: 1) pela inclusão de ectoderma não-neuronal durante a embriogênese; 2) como resposta tecidual no curso de uma lesão; 3) por reação à presença de um corpo estranho; ou 4) por indução iatrogênica durante procedimentos cirúrgicos. Sendo assim, pode ter origem congênita ou adquirida (Andrade et al., 2012; Featherstone \& Llabres Diaz, 2003). \section{B] M Brazilian Journal of Veterinary Medicine \\ p-ISSN 0100-2430 e-ISSN 2527-2179

Como citar: Silva, B. R. S. A, Santos Filho, M. Bendas, A. J. R., Soares, D. V., Mattos, R. G. M. R. B., Suzano, S. M. C., \& Paiva J. P. (2017). Obstrução de via aérea superior secundária a cisto epidermóide laringiano em gato: relato de caso. Brazilian Journal of Veterinary Medicine, 39(1), 74-81. doi: 10.29374/2527-2179.bjvm887

Fonte de financiamento: BRSAS e MSF são bolsistas CAPES; AJRB é bolsista CNPq.

Conflito de interesses: Os autores declaram não haver conflito de interesses que precisam ser informados.

Recebido: Março 13, 2017.

Aceito: Agosto 12, 2017.

O estudo foi realizado no Instituto de Especialidades em Medicina Veterinária, Rio de Janeiro, RJ, Brasil.

\section{*Correspondência}

Bruno Ricardo Soares Alberigi da Silva Instituto de Veterinária, Universidade Federal Rural do Rio de Janeiro - UFRRJ

BR 465, Km 7, Campus Universitário, Bairro Zona Rural

CEP 23897-000 - Seropédica (RJ), Brasil

E-mail: bruno.alberigi@gmail.com

Copyright Silva et al. Este é um

artigo publicado em acesso aberto (Open Access) sob a licença Creative Commons Attribution Non-Commercial, que permite uso, distribuição e reprodução em qualque meio, sem restrições desde que sem fins comerciais e que o trabalho original seja corretamente citado. 
Quando presente na laringe, este tipo de cisto se caracteriza por uma cavidade fechada, geralmente localizada no terço médio da prega vocal, na camada superficial da lâmina própria e no ligamento vocal. Esta cavidade é revestida por epitélio escamoso estratificado e constantemente ligada a fibras elásticas ou colagenosas do ligamento vocal (Gadelha et al., 1998).

As afecções que acometem a laringe acarretam alterações na entrada e na saída do fluxo de ar e na fonação. Os principais achados clínicos de disfunção laringiana incluem: dispneia, ruídos inspiratórios e estridores, disfagia, vômitos e em casos agudos de laringite, tosse (Vadillo, 2007).

Conforme o grau do acometimento do órgão, podem ocorrer sinais clínicos mais graves em consequência do processo obstrutivo. A dispneia, trabalho respiratório para manutenção das pressões arteriais parciais de oxigênio e dióxido de carbono $\left(\mathrm{PaO}_{2}\right.$ e $\mathrm{PaCO}_{2^{\prime}}$ respectivamente), reflete nestas afecções, a dificuldade do animal em obter a fração inspirada de oxigênio $\left(\mathrm{FIO}_{2}\right)$ necessária para suprir a demanda de oxigênio basal para manutenção do organismo (Manchado \& Masián, 2007).

Para o diagnóstico das doenças da laringe é realizada à inspeção direta do órgão e de sua luz por laringoscopia, avaliando-se a movimentação das cartilagens aritenóides e presença de hiperemia, secreções ou formações em seu interior (Merlo \& Real, 2007; Macphail, 2014). Exames complementares como radiografia, ultrassonografia, e tomografia computadorizada podem auxiliar na detecção de lesões menores, que passem despercebidas à inspeção do órgão, ou que estejam localizadas em regiões de difícil identificação ao exame direto da laringe (Carlisle et al., 1991; Rudorf \& Barr, 2002).

O tratamento recomendado para formações benignas de laringe é a sua remoção cirúrgica (Merlo \& Real, 2007). Quando o procedimento cirúrgico não é possível, a abordagem clínica recomendada é baseada no controle da inflamação e consequências do processo obstrutivo, como uso de corticosteroides, mucolíticos e antibióticos quando houver sinais de infecção oportunista (Gadelha et al., 1998; Holt, 2004; Merlo \& Real, 2007).

O presente trabalho tem como objetivo relatar um caso de cisto epidermóide na laringe, causando obstrução de via aéreas superiores em um gato, descrevendo seus achados de exame físico e de exames de imagem como apoio diagnóstico.

\section{Histórico}

Foi atendido um felino, fêmea, sem raça definida (S.R.D) de oito anos, em quadro de angustia respiratória, com histórico de dispneia inspiratória e expiratória. Na anamnese, o tutor informou presença de perda de apetite e emagrecimento progressivo. O paciente recebia corticosteroide por via inalatória (fluticasona) para tratamento de doença brônquica felina há alguns anos.

No exame físico, os parâmetros vitais se encontravam sem alterações. À inspeção o paciente apresentava escore de condição corporal 2/5 (Edney \& Smith, 1986) e foi observado presença de dispneia inspiratória e expiratória. À auscultação cardíaca não foram evidenciadas alterações nas bulhas e silêncios. Durante a auscultação pulmonar constatou-se estridor inspiratório e aumento do ruído expiratório. A percussão torácica se apresentava hiperssonora em todos os campos pulmonares. Na palpação traqueal o animal apresentou sensibilidade em região cranial da traqueia. Em função do quadro de dispneia, foi introduzida oxigenioterapia e o paciente foi sedado com meperidina ( $3 \mathrm{mg} / \mathrm{kg} / \mathrm{IM})$.

Após estabilizado o quadro de dispneia, o animal foi submetido a radiografia torácica e cervical nas posições latero-lateral e ventrodorsal. Por meio da radiografia torácica foi visualizado aumento de densidade dos campos pulmonares acarretado por padrão predominantemente bronquial, acompanhado de bronquiectasia de distribuição difusa e silhueta cardíaca sem alterações. Foi possível identificar aumento da distância entre a silhueta cardíaca e a cúpula diafragmática (Figura 1 e2), associada a retificação do diafragma (Figura 1) e presença de protuberâncias diafragmáticas (Figura 2). Foi percebido ainda, dilatação esofágica e presença de grande quantidade de gás em estômago e alças intestinais (Figura 1). Na radiografia da região cervical, foi visualizada estrutura ovalada de média radiopacidade, medindo cerca de $2,6 \mathrm{~cm}$ em seu maior eixo, sobrepondo topografia da laringe (Figura 3).

Com intuito de obter melhor informação sobre o diagnóstico, foi realizado exame ultrassonográfico da região cervical, onde foi notado estrutura ovalada, com ecotextura grosseira e hipoecóica, medindo cerca de $2,0 \mathrm{~cm}$ em seu maior eixo identificado, sobrepondo a porção direita das 


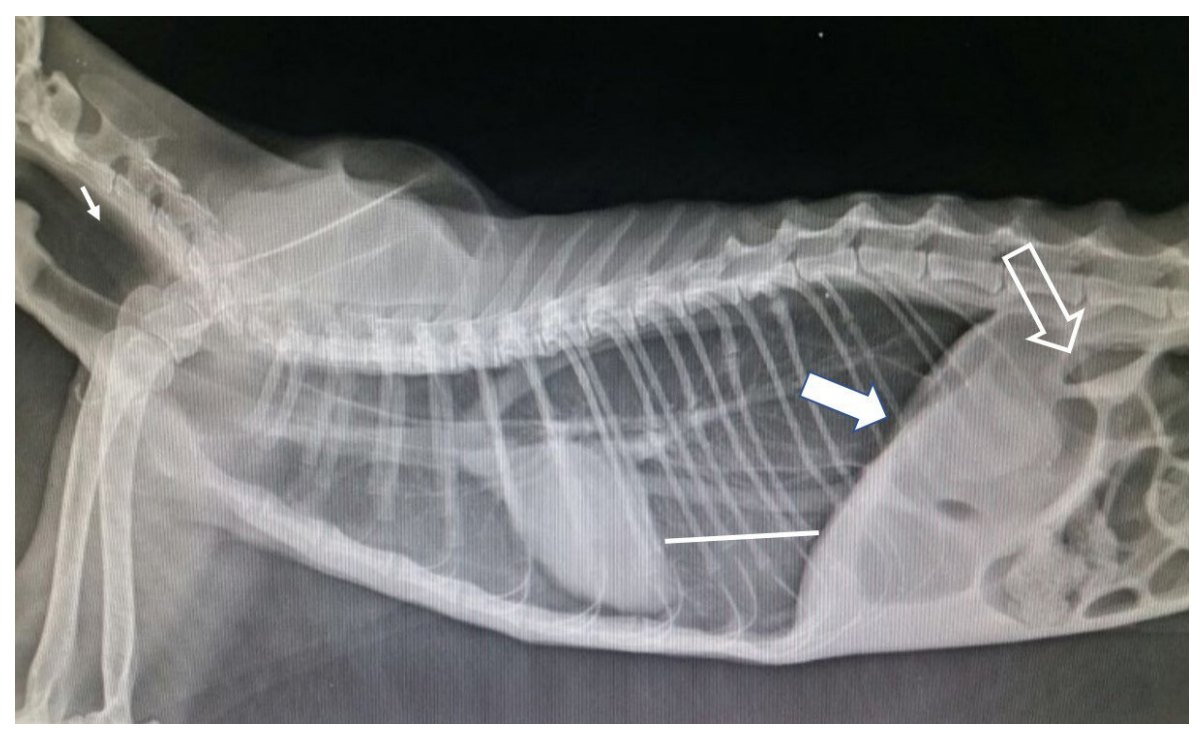

Figura 1. Imagem radiográfica de tórax do paciente relatado, na posição latero-lateral, evidenciando retificação do diafragma (seta cheia), aumento da distância da silhueta cardíaca e cúpula diafragmática (linha branca), dilatação esofágica (ponta de seta) e grande quantidade de gás em estômago ealças intestinais (contorno de seta).

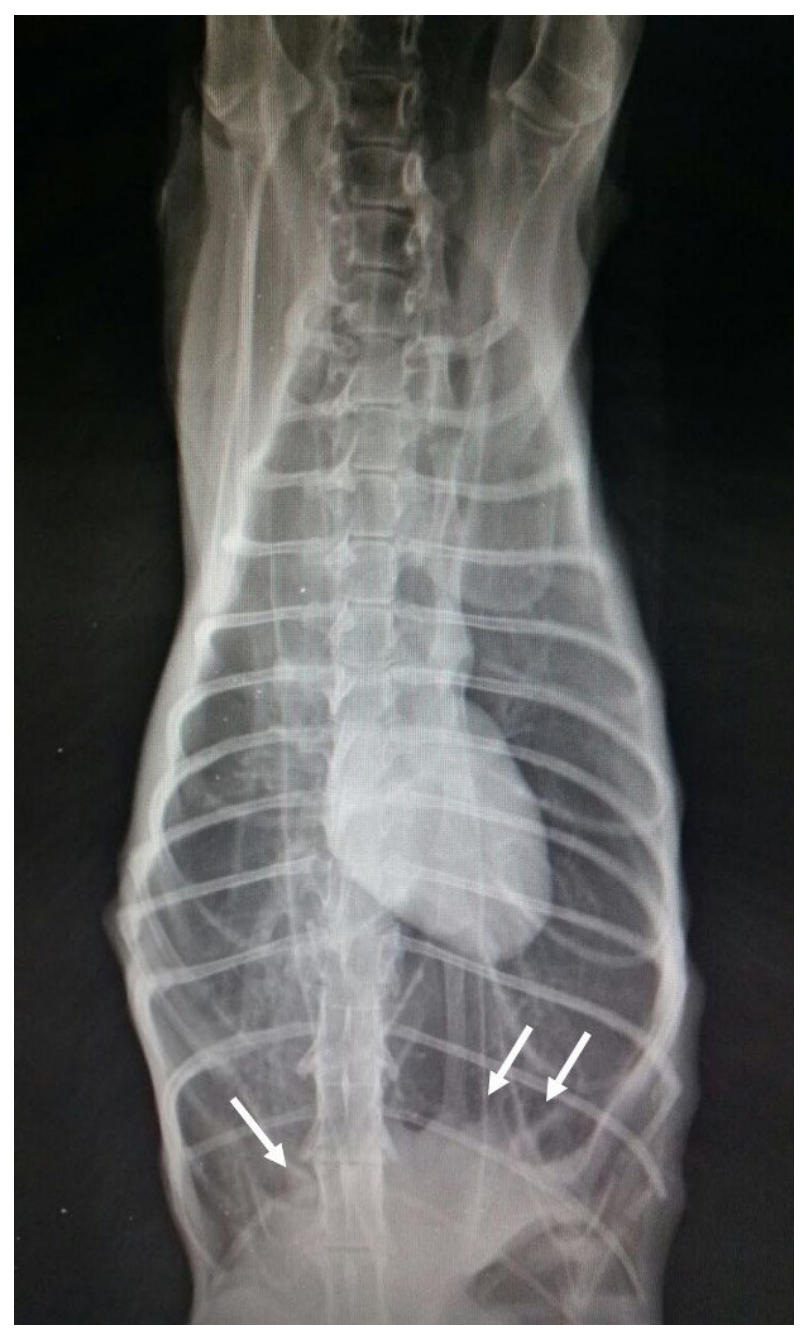

Figura 2. Imagem radiográfica de região torácica na posição ventro-dorsal do paciente relatado, evidenciando aumento da distância da silhueta cardíaca e cúpula diafragmática e presença de protuberâncias diafragmáticas (setas). 
cartilagens laríngeas, se estendendo para a região da rima da glote e aparentemente comprimindo a mesma no momento do exame.

Foi informado ao tutor, sobre as possibilidades diagnósticas, como neoplasia laringiana, e o mesmo optou pela eutanásia, visto o prognóstico desfavorável para um animal de oito anos de idade. Após a eutanásia e autorização do tutor, realizou-se a necropsia.

Durante a necropsia, após a retirada da traquéia e laringe foi notada estrutura de tonalidade esbranquiçada medindo 1,5 cm de diâmetro deslocando ventral da cartilagem epiglotee compressão das cartilagens aritenóides (Figura 4 e 5). Não foram identificadas massas ou alterações significativas

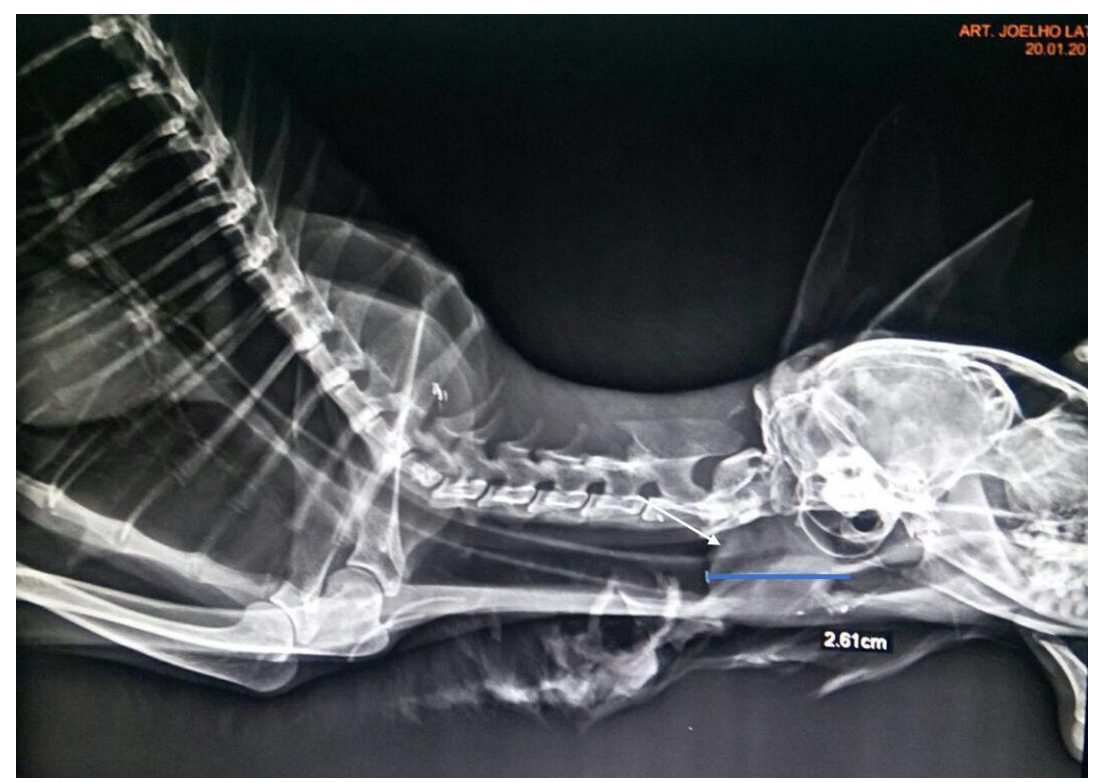

Figura 3. Exame radiográfico da região cervical do paciente relatado, na posição latero-lateral, evidenciando estrutura oval (seta) de média radiopacidade, medindo cerca de 2,6 cm em seu maior eixo, sobrepondo topografia da laringe.

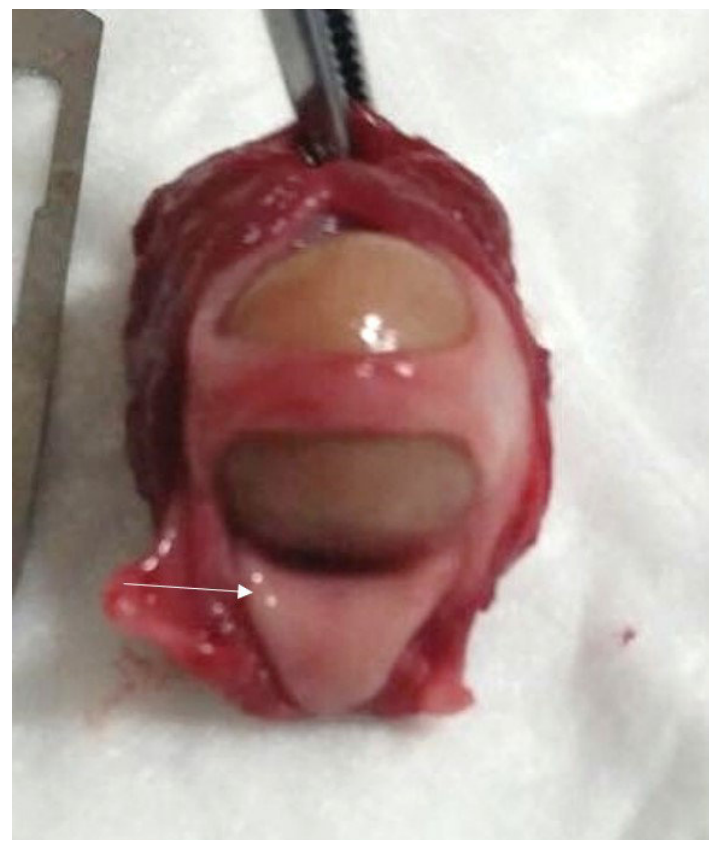

Figura 4. Fragmento de laringe evidenciado, massa de grandes dimensões deslocando ventralmente a cartilagem epiglote (seta). 
em outros órgãos que sugerissem outros focos neoplásicos. O conjunto traquéia e laringe junto com a massa em seu interior foi fixado em formol a 10\% e enviado para análise histopatológica.

No exame histopatológico foi observado tecido revestido por epitélio escamoso, apresentando lesão cística em lâmina própria, revestida em seu interior por camada delgada de epitélio escamoso, sem atipias. No lúmen da lesão cística observou-se abundante quantidade de material queratinoso (Figura 6 e 7). Não foram observados indícios de malignidade nas secções avaliadas, sendo os achados histológicos compatíveis com cisto epidermóide.

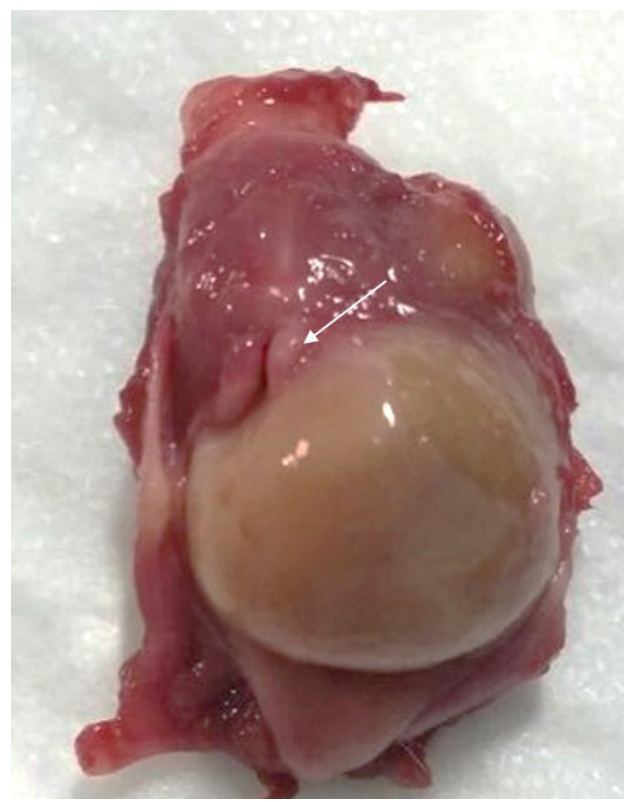

Figura 5. Fragmento de laringe, durante procedimento de necropsia, evidenciando massa de grandes dimensões causando compressão (seta) das cartilagens aritenóides.

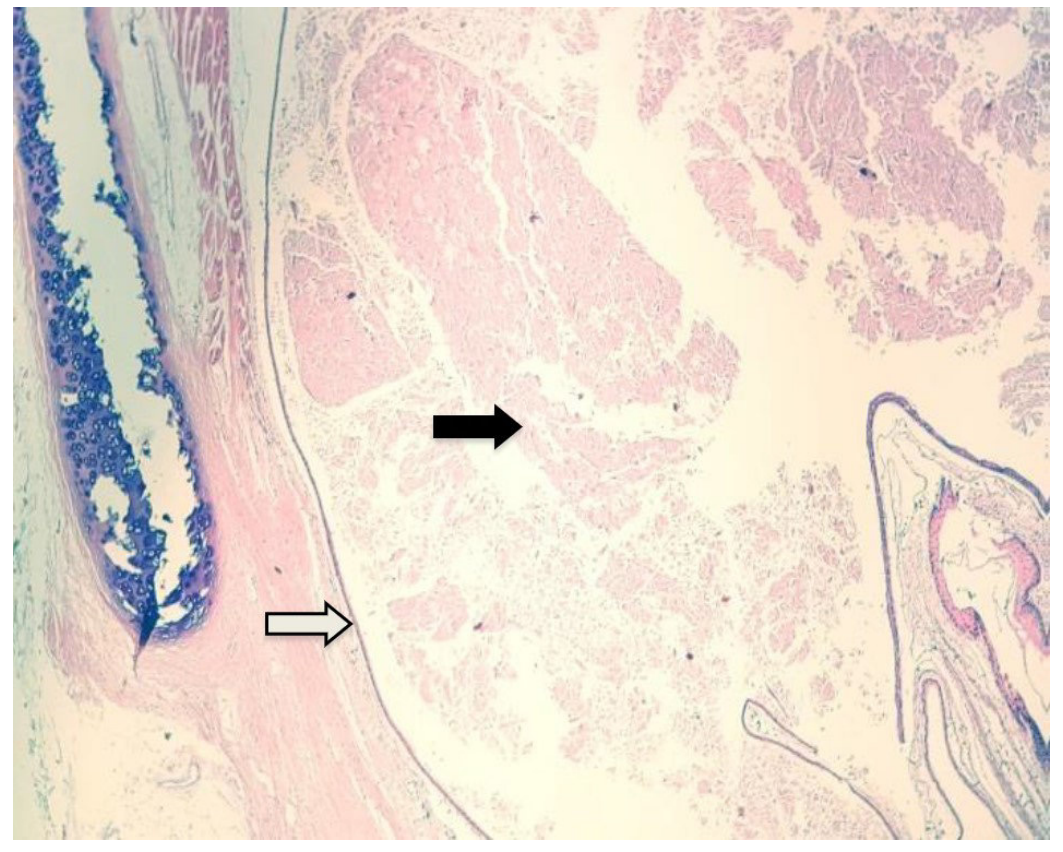

Figura 6. Fotomicrografia de cisto epidermóide em laringe. Lesão cística revestida por epitélio escamoso (seta branca) contendo aglomerados de queratina (seta preta). Hematoxilina e eosina. Objetiva de 04X. Fonte: Histopet. 


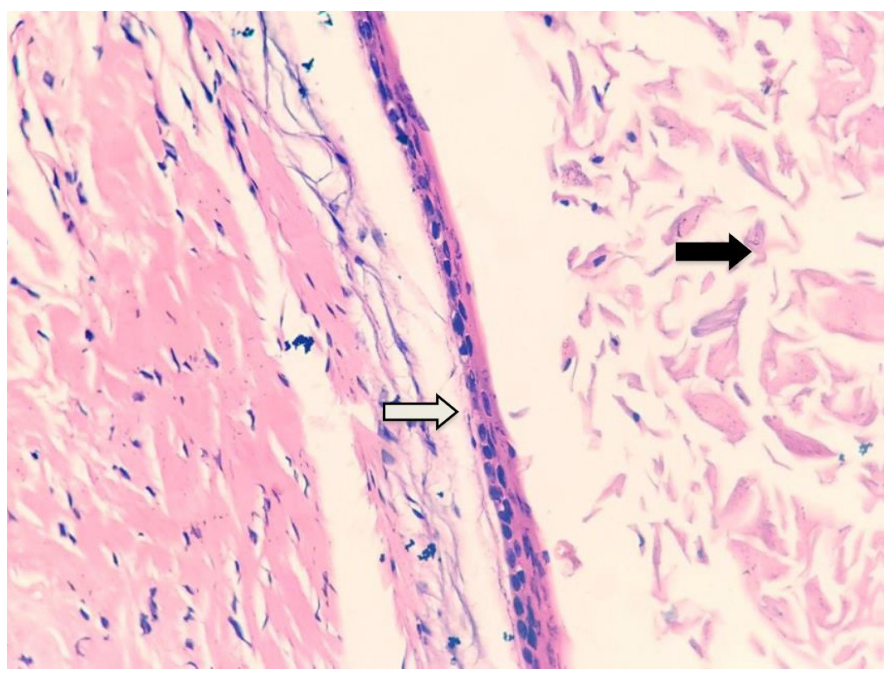

Figura 7. Fotomicrografia de cisto epidermóide em laringe. Lesão cística revestida por epitélio escamoso (seta branca) contendo aglomerados de queratina (seta preta). Hematoxilina e eosina. Objetiva de 40X. Fonte: Histopet.

\section{Discussão}

O presente relato trata de uma rara afecção laringiana em felinos, com escassa descrição em literatura (Taylor et al., 2009; Macphail, 2014). O seu plano diagnóstico envolve a reunião de todos os achados coletados no exame clínico que direcionarão para suspeita clinica de obstrução de vias aéreas anteriores (Corgozinho et al., 2008).

Os achados de histórico e anamnese são inespecíficos e o emagrecimento provavelmente ocorreu em função da perda de apetite causada pela dispneia e a provável dificuldade na deglutição pela presença da massa de grande dimensão na referida topografia (Vadillo, 2007). O histórico de doença brônquica felina, pode ter mascarado a percepção da evolução da doença, uma vez que o entendimento da dispneia e sua classificação, que auxiliarão na localização da doença dentro do sistema respiratório, requer experiência direcionada para afecções respiratórias, representando desafio diagnóstico.

Foi observado no paciente relatado presença de dispneia mista à inspeção. Esta dispneia é caracterizada pelo aumento do trabalho respiratório nos dois momentos do ciclo (inspiração e expiração). A dispneia inspiratória foi identificada pela visualização do aumento do trabalho muscular, gerando movimento toraco-abdominal parodoxal associado ao prolongamento da inspiração (Manchado \& Masián, 2007). Este quadro é conhecido como esforço inspiratório e facilmente identificado pelo clínico, representando um importante achado na suspeita clínica de obstrução de vias aéreas anteriores.

A dispneia expiratória, com apresentação mais sutil, foi identificada ao se constatar o aumento do tempo expiratório sugerindo a existência de obstrução em pequenas vias aéreas, que gera a necessidade de um maior esforço para expulsão do ar (Bay \& Johnson, 2004; Trzil \& Reinero, 2014). Nesta dispneia é percebido o recrutamento de musculatura abdominal expiratória não atuantes em situações fisiológicas, mas frequentemente observado em animais com doença brônquica (Bay \& Johnson, 2004; Manchado \& Masián, 2007).

O estridor detectado a ausculta, é um ruído característico produzido por obstrução laringiana (Manchado \& Masián, 2007), e sua origem está no fluxo turbulento de ar produzido na passagem deste pela obstrução (Holt, 2004). O aumento de ruídos expiratórios ocorre em função da obstrução das pequenas vias aéreas dificultando a saída do ar no momento da expiração como em casos de asma brônquica, enfisema e bronquite (Holt, 2004; Manchado \& Masián, 2007; Vadillo, 2007).

Não se pode deixar de considerar a contribuição da obstrução laringiana na ocorrência da dispneia expiratória. O fechamento fisiológico das cartilagens aritenoides no momento da expiração é o responsável pelo tempo maior deste momento do ciclo respiratório, mecanismo importante para o recrutamento alveolar (Hendricks, 2004), desta forma é possível associar que 
processos obstrutivos laringianos, fechando ainda mais sua luz durante a expiração, contribuam para a ocorrência da dispneia expiratória (Corgozinho et al., 2008).

A percussão, juntamente com a auscultação, é indicada para avaliação do tórax, pois permite delimitar os campos pulmonares e detectar aumento e diminuição de ar nos alvéolos (Manchado \& Masián, 2007). No presente relato a percussão revelou som hiperssonoro, indicando assim um estado de hiperinsuflação pulmonar, devido à dificuldade dos alvéolos em expulsarem o ar, por conta da obstrução mecânica das pequenas vias aéreas característica da doença brônquica felina (Bay \& Johnson, 2004; Manchado \& Masián, 2007).

O aumento da sensibilidade a palpação da região cranial da traqueal, permitiu junto com outros achados do exame clínico como a dispneia inspiratória, aproximar o foco da afecção, contudo sem ainda saber o tipo de alteração presente.

Em função da angustia respiratória, o paciente foi submetido a oxigenoterapia e sedado com meperidina como fármaco tranquilizante para permitir uma oxigenoterapia adequada para aumento da $\mathrm{FIO}_{2}$ (Costello et al., 2001). A tranquilização diante de quadros de dispneia, é amplamente recomendada na literatura, pois a agitação é um importante fator agravante deste quadro (Macphail, 2014).

Os achados da radiografia torácica como: 1) aumento da distância do coração em relação ao diafragma; 2) retificação diafragmática; 3) aumento da área pulmonar e 4) presença de múltiplas protuberâncias no diafragma, que representam os locais de fixação do diafragma nas costelas, convergem para o estado de hiperinsuflação pulmonar (Thrall, 2013). Este estado não deve ser apenas associado ao aprisionamento de ar decorrente da obstrução das pequenas vias aéreas pela doença brônquica, mas também ao quadro de obstrução laringiana que compromete não apenas a inspiração como também a expiração.

A dilatação esofágica assim como a distensão gástrica e de alças intestinais por gás, estavam presentes devido aerofagia secundária a dispneia (Bichards \& Sherding, 2008).

Na radiografia cervical, observou-se presença de estrutura ovalada de média radiopacidade que poderia ser a causa da obstrução, neste momento foi indicado a ultrassonografia cervical (Rudorf et al. 1999), devido a fácil disponibilidade do exame, sendo este exame sensível para detecção de alteração na orofaringe e minimamente invasivo para pacientes críticos (Rudorf \& Barr, 2002).

Diante dos achados clínicos que direcionaram para um quadro obstrutivo de vias aéreas anteriores (Corgozinho et al., 2008; Vadillo, 2007; Macphail, 2014), associados a visualização da estrutura ovalada em topografia de laringe estabeleceu a suspeita clínica de neoplasia. Sendo assim, em função da grave manifestação clínica, idade do paciente, dimensão e localização da estrutura evidenciada nos exames de imagem, que se traduziria em procedimento cirúrgico de alta complexidade e um pós-operatório delicado, o tutor optou pela eutanásia.

Diante do encaminhamento da eutanásia, não foi realizada a laringoscopia in vivo, pois necessitaria de aprofundamento anestésico e a mesma foi postergada para o momento da necropsia.

A realização da necropsia possibilitou a confirmação da doença obstrutiva de via aérea anterior, mais precisamente, doença obstrutiva laringiana sem, no entanto, alcançar ao diagnóstico definitivo. A ausência de neoformações em outros órgãos descartou a condição metastática macroscópica, caso o diagnóstico fosse neoplasia maligna.

Os achados histopatológicos concluíram que a estruturas e tratava de um cisto epidermóide. Inicialmente a suspeita clínica baseada nos exames de imagem foi de neoplasia laringiana, que apesar de rara em gatos já foi descrita algumas vezes na literatura (Merlo \& Real, 2007; Taylor et al., 2009; Macphail, 2014).

A histopatologia demonstra no presente relato que nenhum outro exame, apesar da história clínica e idade do paciente, a substitui, e caso o quadro clínico fosse identificado mais precocemente, quando a estrutura ainda tivesse dimensões menores e houvesse menor comprometimento do estado geral do paciente, o caso clínico poderia ter obtido êxito no tratamento cirúrgico.

Evidencia-se a importância do posicionamento profissional do clínico veterinário, solicitando a realização da necropsia diante de um caso que facilmente permaneceria com o diagnóstico presuntivo de câncer. Desta forma, foi possível alcançar o diagnóstico definitivo de afeç̧ão de ocorrência mais rara, mais que não deve deixar de compor o rol do diagnóstico diferencial. 


\section{Conclusão}

O presente relato demonstra como a investigação de afecções das vias aéreas devem ser amplamente estudadas e detalhadas pelo clínico, não podendo ser descartada outras possíveis suspeitas na presença de uma afecção inicial que justifique alguns sintomas. No presente relato os achados do exame clínico e exames complementares anteriores, direcionaram o tratamento para doença brônquica. Porém, outros achados de um exame clínico mais apurado, como a classificação da dispneia e ausculta detalhada, poderiam ter direcionado para exames diagnósticos mais adequados, e consequentemente possibilitar melhor conduta terapêutica antes do quadro clinico evoluir para estado grave.

\section{Referências}

Andrade, R. L. S., Oliveira, D. M., Dantas, A. F. M., Souza, A. P., Nóbrega Neto, P. I., \& Riet-Correa, F. (2012). Tumores de cães e gatos diagnosticados no semiárido da Paraíba. Pesquisa Veterinária Brasileira, 32(10), 1037-1040. http://dx.doi.org/10.1590/s0100-736X2012001000016.

Bay, J. D., \& Johnson, L. R. (2004). Feline bronchial disease/asthma. In King L. G. (Ed.), Textebook of respiratory disease in dogs and cats (pp. 388-396). Missouri: Saunders.

Bichards, S. J., \& Sherding, R. G. (2008). Manual Saunders: clínica de pequenos animais (3a ed., 2072 p.). São Paulo: Roca.

Carlisle, C. H., Biery, D. N., \& Thrall, D. E. (1991). Thracheal and laryngeal tumors in the dog and cat: literature review and 13 additional patients. Veterinary Radiology \& Ultrasound, 32(5), 229-235. http://dx.doi. org/10.1111/j.1740-8261.1991.tb00112.x.

Corgozinho, K. B., Souza, H. J. M., Ferreira, A. M. R., Cunha, S. C., \& Damico, C. (2008). Dispnéia provocada por tumor laringeano em gatos no período de 1997 e 2007. Acta Scientiae Veterinariae, 36(3), 289-292.

Costello, M. F., Keith, D., Hendrick, M., \& King, L. (2001). Acute upper airway obstruction due to inflammatory laryngeal disease in 5 cats. Journal of Veterinary Emergency and Critical Care, 11(3), 205-210. http://dx.doi. org/10.1111/j.1476-4431.2001.tb00085.x.

Edney, A. T., \& Smith, P. (1986). Study of obesity in dogs visiting veterinary practices in the United Kingdom. The Veterinary Record, 118(14), 391-396. http://dx.doi.org/10.1136/vr.118.14.391. PMid:3716092.

Featherstone, H., \& Llabres Diaz, F. (2003). Maxillary bone epithelial cyst. The Journal of Small Animal Practice, 44(12), 541-545. http://dx.doi.org/10.1111/j.1748-5827.2003.tb00118.x. PMid:14692552.

Gadelha, M. E. C., Gonçalves, M. I. R., \& Pontes, P. A. L. (1998). Alterações estruturais mínimas da laringe. In Pinho S. M. R. (Ed.), Fundamentos em fonoaudiologia: tratando os distúrbios da voz (pp. 65-71). São Paulo: Guanabara Koogan.

Hendricks, J. C. (2004). Respiratory muscle fatigue and failure. In King L. G. (Ed.), Textebook of respiratory disease in dogs and cats (pp. 61-65). Missouri: Saunders.

Holt, D. E. (2004). Upper airway obstruction, stertor, and stridor. In: King L. G. (Ed.), Textebook of respiratory disease in dogs and cats (pp. 35-42). Missouri: Saunders.

Macphail, C. (2014). Laryngeal disease in dogs and cats. The Veterinary Clinics of North America. Small Animal Practice, 44(1), 19-31. http://dx.doi.org/10.1016/j.cvsm.2013.09.001. PMid:24268331.

Manchado, J. É., \& Masián, D. S. (2007). História clínica e avaliação física do paciente respiratório. In J. A. M. Alonso (Ed.), Enfermidades respiratórias em pequenos animais (pp. 3-9). São Paulo: Interbook.

Merlo, E. M. M., \& Real, I. G. (2007). Tumores de laringe. In Alonso, J. A. M. (Ed.), Enfermidades respiratórias em pequenos animais (pp. 211-214). São Paulo: Interbook.

Rudorf, H., \& Barr, F. (2002). Echolaryngography in cats. Veterinary Radiology \& Ultrasound: the Official Journal of the American College of Veterinary Radiology and the International Veterinary Radiology Association, 43(4), 353-357. PMid:12175000.

Rudorf, H., Lane, J. G., Brown, P., \& Mackay, A. (1999). Ultrasonographic diagnosis of a laryngeal cyst in a cat. The Journal of Small Animal Practice, 40(6), 275-277. http://dx.doi.org/10.1111/j.1748-5827.1999.tb03079.x. PMid:10404488.

Taylor, S. S., Harvey, A. M., Barr, F. J., Moore, A. H., \& Day, M. J. (2009). Laryngeal disease in cats: a retrospective study of 35 cases. Journal of Feline Medicine and Surgery, 11(12), 954-962. http://dx.doi.org/10.1016/j. jfms.2009.04.007. PMid:19539507.

Thrall, D. E. (2013). The canine and felinelung. In: E. Donald \& D. E. Thrall (Eds), Textebook of veterinary diagnostic radiology (6a ed., pp. 608-631). Missouri: Elsevier.

Trzil, J. E., \& Reinero, C. R. (2014). Update on feline asthma. The Veterinary Clinics of North America. Small Animal Practice, 44(1), 91-105. http://dx.doi.org/10.1016/j.cvsm.2013.08.006. PMid:24268335.

Vadillo, A. C. (2007). Síndrome braquicefálica e paralisia laríngea em cães. In: Alonso J.A.M. (Ed.), Enfermidades respiratórias em pequenos animais (pp. 93-98). São Paulo: Interbook. 